\title{
Acoustic startle reactions, activity, and background noise intensity, before and after lesions of medial cortex in the rat
}

\author{
JAMES R. ISON and LAURA SILVERSTEIN \\ University of Rochester, Rochester, New York 14627
}

\begin{abstract}
Acoustic startle reactivity and stabilimetric activity at five background noise levels, from 30 to $90 \mathrm{~dB}$ (SPL), were assessed in five rats. A typical biphasic function for the startle reflex resulted as amplitudes first increased with more intense noise levels to an optimal value and then declined. Activity was an increasing linear function of background noise level. The midline cortex was then ablated from the frontal poles to the level of the crossing of the anterior commissures. In a posttest, overall startle reactivity was increased but the biphasic reflex function was maintained. Activity measures did not change. Evidently, frontal midline cortex is not a necessary part of the forebrain system subserving the arousing consequence of background noise level.
\end{abstract}

In the rat, the relationship between the vigor of the acoustic startle reflex and the ambient noise level is biphasic (Ison \& Hammond, 1971). Up to a moderate level, the background noise has a tonic invigorating effect and the reflex is facilitated by the greater noise level. The optimal point is in the approximate range of $60.75 \mathrm{~dB}$ (SPL), and further increases in ambient noise thereafter have a deleterious effect. The increasing early part of the function may reflect an arousal consequence of the noise, a consequence which may be noted similarly in a variety of reflexive and nonreflexive behaviors (for example, Ison \& Leonard, 1971; MacMillan, Gray, \& Ison, 1973; Sheard, Astrachan, \& Davis, 1975). The reason for the terminal downturn is not unambiguous but it may be produced by the increasingly unfavorable signal/noise ratio as the background level noise approaches the intensity of the eliciting stimulus signal (Ison \& Hammond, 1971). Thus, for example, Davis (1974) showed that the optimal background level varied sensibly with the intensity of the eliciting stimulus, occurring at a more intense level with the more intense elicitor.

In subsequent work, Davis and Gendelman (1977) have concluded that the ascending limb representing the hypothetical arousal process has its anatomical substrate in the forebrain of the rat. A decerebrate rat preparation was achieved by a knife-cut transection running from the retrosplenial cortex through to the base of the brain just posterior to the mammillary bodies. These animals were no different from intact

This research was supported by a research grant from the National Institute of Neurological and Communicative Disorders and Stroke, NS-12443. The authors thank G. J. Thomas and J. Conlon for their technical help. Reprints may be obtained from James R. Ison, Department of Psychology, Psychology Building, University of Rochester, Rochester, New York 14627. rats in showing temporal recovery function or stimulusproduced inhibition. However, they did not exhibit reflex habituation with repeated stimulation, and they did not show the usual facilitation of the startle with an increase in ambient noise level.

The question in the present exploratory study was whether the frontal cortex might be implicated in producing this consequence of decerebration. There are pathways from the reticular formation through the nonspecific thalamic nuclei to the frontal cortex (Skinner \& Lindsley, 1967; Velasco \& Lindsley, 1965) and from the frontal cortex directly to the reticular formation (Clemente, 1968), which are intimately involved with arousal and behavioral inhibition. It is possible, then, that the frontal cortex may be a rostral component of the system subserving startle reflex activation, a system which is severed in the decerebrate animal.

Previous work in our laboratory (Hammond, 1974) has shown that lesions in the frontal medial cortex result in an enhanced startle reflex compared either with preoperative behavior or with the postoperative behavior of sham controls. In the earlier work, the background was stable at approximately the optimal value $(70 \mathrm{~dB})$. In the present experiment, animals received a pretest in which the acoustic startle reflex was elicited in a range of background intensities intended to sample on both sides of the optimal value. The amount of spontaneous activity at these several intensities was also assessed. Surgery intended to destroy the midline cortex anterior to the coordinates of the anterior commissure was performed on all animals and followed by a posttest. The experimental question of primary interest was whether the function relating startle reflex amplitudes to the background noise level would maintain its typically biphasic appearance following the surgical intervention. 


\section{METHOD}

\section{Subjects}

Five male albino rats were used, weighing from $300-350 \mathrm{~g}$ at the time of surgery. They were obtained from the Holtzman Co., Madison, Wisconsin. Except for the day preceding surgery, food and water were available ad lib.

\section{Apparatus}

The animals were run in a small cage $(16 \times 7 \times 9.5 \mathrm{~cm})$ with brass-rod sides and a floor made of two steel plates separated by a midline gap of $1.2 \mathrm{~cm}$. The cage was held firmly in a frame by compression springs. A white-noise background was provided by a Grason-Stadler white-noise generator, the output of which passed through a Daven attenuator network to DLK cross-over speakers adjusted to give a flat output from about .1 to $10 \mathrm{kHz}$. The startle stimulus was provided by a HewlettPackard oscillator delivered to a Lansing high-frequency tweeter. It went through an electronic switch controlled by electronic timers, was amplified, then passed through a terminal relay circuit which was closed only during stimulus delivery. Stimulus intensities were measured on a General Radio soundlevel meter and impact analyzer and are reported with respect to a baseline of $20 \mu \mathrm{N} / \mathrm{m}^{2}$.

An accelerometer mounted on the cage detected the animal's reaction to the acoustic stimuli. The output of the accelerometer was amplified on a Grass polygraph, and fed to a storage CRT which was triggered at the onset of the startle stimulus. The size of the ensuing reaction was recorded as millimeter deflection of the largest peak within $50 \mathrm{msec}$ of the stimulus. The amplifier output was also fed into an integrator which allowed measurement of stabilimetric activity during the imposition of the different background conditions. For all data analysis, the grand total of each animal's response amplitudes was set at 100 , and then the totals within each condition were expressed as a proportion of that value. These normalized relative response amplitudes have the advantage of weighting each animal's contribution equally in the analyses; otherwise, animals with larger overall scores are more heavily weighted.

The animal cage and stimulus devices were placed in a singlewalled IAC sound-attenuating room. Stimulus control, timing gear, and the recording apparatus were in a nearby room. The animal was watched over closed circuit TV.

\section{Procedure}

The animal was placed in the cage in the absence of any imposed background for several minutes. It then received a series of five "warm-up" trials, one at each of five background conditions. The background conditions were ambient room level (at $27-28 \mathrm{~dB}$, labeled nominally as $30 \mathrm{~dB}$ ), and four levels of imposed white noise at $45,60,75$, and $90 \mathrm{~dB}$. The startle stimulus was a $25-\mathrm{msec}, 110-\mathrm{dB}, 10-\mathrm{kHz}$ tone, with rise-decay times of $5 \mathrm{msec}$. The procedure was to turn on a particular background condition for at least $30 \mathrm{sec}$, which was the time unit for integrated activity. At the end of $30 \mathrm{sec}$, the startle stimulus was given as soon as the animal was sitting quietly and was oriented in the cage along the midline. The background noise was then turned off for 5-10 sec, and this was followed by the next condition. After the five "warm-up" trials, the animal received a series of 30 trials, each condition being given in random sequence within each of six blocks of 5 trials.

Surgery was performed approximately 2 weeks after the pretest (range 10-19 days). The animals were given $1.6 \mathrm{mg}$ of atropine (IP) and $10 \mathrm{~min}$ later sodium pentobarbital, following Valenstein (1961). All of the animals received multiple anodal electrolytic lesions intended to fuse and destroy the medial cortex of the frontal poles and the midline supra callosal cortex back to about the level of the midline crossing of the anterior commissure. The surgical procedures are described in Barker and Thomas (1965). Briefly, a series of five pairs of holes, 1 to

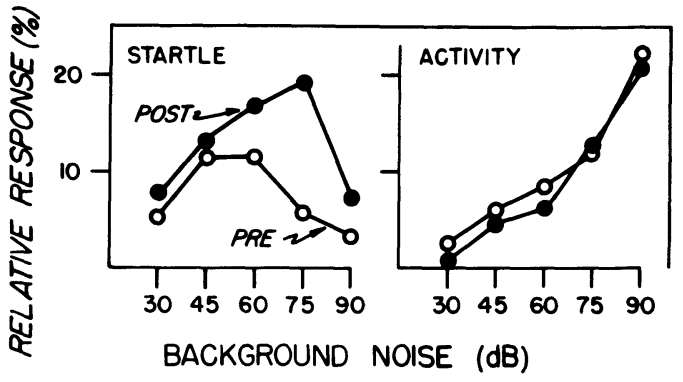

Figure 1. Relative startle amplitudes and relative activity scores before and after surgery at each of the five background intensities.

$1.5 \mathrm{~mm}$ apart across the midline ran from 1.2 to $4.8 \mathrm{~mm}$ anterior to bregma. Stainless steel electrodes, dropped at each site to progressively deeper positions, were used, with $1.5 \mathrm{~mA}$ of current passed for $15 \mathrm{sec}$ at each placement.

About 2 weeks following surgery (range 11-17 days), each animal received a posttest which was identical to the pre-test in all respects. Two weeks later, they were deeply anesthetized with sodium pentobarbital and perfused through the heart with $4 \%$ buffered Formalin. The brains were removed from the skulls and, after further hardening, were embedded in celloidin and sectioned in the frontal plane at $48 \mu$. Every third section was stained with cresyl violet.

\section{RESULTS AND DISCUSSION}

Figure 1 depicts the relative startle magnitudes and the relative activity scores at each of the background noise levels before and after surgery. In the preoperative data, the typically biphasic curve relating startle amplitudes to noise levels is most apparent, the function having its peak at $60 \mathrm{~dB}$. In contrast, activity increased steadily with the increase in background noise. The increasing portion of these curves is typically ascribed to a tonic-arousing consequence of white noise, and the declining portion to the increasing similarity in the intensities of the startle "signal" and the background "noise" (Davis, 1974; Ison \& Hammond, 1971). Although the hypothesis of sensory masking seems plausible, subtleties in the function, considered in light of the actual psychophysical data on sensory masking (Stevens, 1966, for example), suggest that it is not a completely adequate explanation of the terminal decrement in the behavioral startle function.

Masking is most effective when the signal is at the same intensity as the mask, and rapidly diminishes as the signal increases in strength; masking has apparently little or no effect on the threshold of the signal once the stimulus exceeds the mask by 20-30 dB. In contrast, its decremental effect on the startle reflex begins to outweigh its facilitative effect when the stimuli are separated by no less than $50 \mathrm{~dB}$. The activity data are of some interest in the illumination of this problem, for they show that, on this behavior, the facilitative effect of the noise continued to increase throughout the range of imposed intensities. To the extent that this linear relationship reflects the growth of the process 
also responsible for the facilitation of startle behavior, it is not possible to attribute any part of the leveling off and subsequent descending limb of the startle function to any leveling off and diminution in arousal. It is then apparent that the interesting point in the nonmonotone reflex function is not the noise level at which the descending limb first becomes evident (beyond $60 \mathrm{~dB}$ ), but the earlier noise level at which the ascending curve deviated from a straight-line increasing function (beyond $45 \mathrm{~dB}$ ). It is simply not plausible that sensory masking of the startle-eliciting signal by the background noise would become effective so early at separations of 50 to $65 \mathrm{~dB}$.

The activity data suggest a second conjecture concerning the descending limb of the startle function. Background noise, because of its tonic activating effect on the motor system, may make the motor system underlying the startle reflex increasingly refractory to punctate input of limited duration. The descending function may then result not because of "sensory masking" of the startle signal but because of a "motor masking" of its normal response consequence.

As for the hypothesis which led to the present research, it was thought that the ascending limb of the startle function might not be apparent in animals after damage to the medial frontal cortex. The postoperative data shown in Figure 1 are unequivocally negative for this hypothesis. The curve relating startle vigor to background intensity remained biphasic, and, indeed, there was a suggestion that the initial ascending portion was more prominent after surgery than before. There was no effect of the surgery on the activity scores. Analysis of variance of the preoperative and postoperative startle data yielded a reliable effect of noise background level $(F=6.89, \mathrm{df}=4 / 16, \mathrm{p}<.005)$ which had a quadratic trend $(F=32.19$, df $=1 / 4$, $\mathrm{p}<.005)$. There was an overall interaction between the noise intensity variable and surgery $(F=4.65, \mathrm{df}=$ $4 / 16, p=.01$ ) which was primarily manifest in a cubic noise trend $(F=12.52, \mathrm{df}=1 / 4, \mathrm{p}<.05)$. The interaction resulted because the facilitative effect of the lesion on the startle amplitude was more apparent in the intermediate noise background (particularly at $75 \mathrm{~dB}$ ) than it was at either less intense or more intense levels. This effect was present in each of the five animals. It is of some interest that the previous data showing a facilitative effect of similar lesions on startle behavior were obtained at an intermediate noise level of $70 \mathrm{~dB}$ (Hammond, 1974). Analyses of activity yielded a significant effect of noise background $(F=21.62$, $\mathrm{df}=4 / 16, p<.001)$ which was primarily exhibited as a linear trend $(F=30.96, d f=1 / 4, p<.005)$. Comparisons of presurgical and postsurgical behaviors yielded no significant effects $(F<1)$. These data may be most similar to those reported by Lynch (1970), who showed no influence of frontal lesions on stabilimetric activity in the satiated rat, although wheel running and activity in the stabilimeter following starvation were exaggerated.
The lack of influence of the surgery on activity may be interpreted as signifying that the arousing effect of noise was not affected by the lesion. From this it follows that the increase in startle behavior seen after the operation does not result from a change in the animal's general arousal level but, instead, from an enhancement in the animal's specific reaction to the eliciting stimulus. The frontal cortex in the intact animal may exert a chronic dampening influence on reflexive behavior, and the increase noted after surgery may then result as a release phenomenon. This is, of course, a classic view of the action of the frontal cortex (as is extensively reviewed by Brutkowski, 1965, for example). The upward shift in the observed peak of the startle function would not be at all anticipated from the notion that nonmonotonicity is produced by sensory masking. It may be consistent with the motor-masking hypothesis on the basis that the more intense motor command might be less affected by the degree to which the motor system is refractory to further input.

Figure 2 shows the extent of the largest and the smallest lesions in three coronal sections, one at the poles, one just rostral to the genu of the corpus callosum, and one just rostral to the crossing of the anterior commissure. The sections are labeled with the AP coordinates at their approximate location in König and Klippel (1963). The animal with the largest lesion was also the animal with the largest postoperative enhancement of the response (a fourfold increase overall with a sevenfold increase at $75 \mathrm{~dB}$ ). The animal with the smallest lesion showed no overall enhancement of the response, although its response at $75 \mathrm{~dB}$ was increased by $233 \%$.

Regardless of the meaning of subtle changes in the

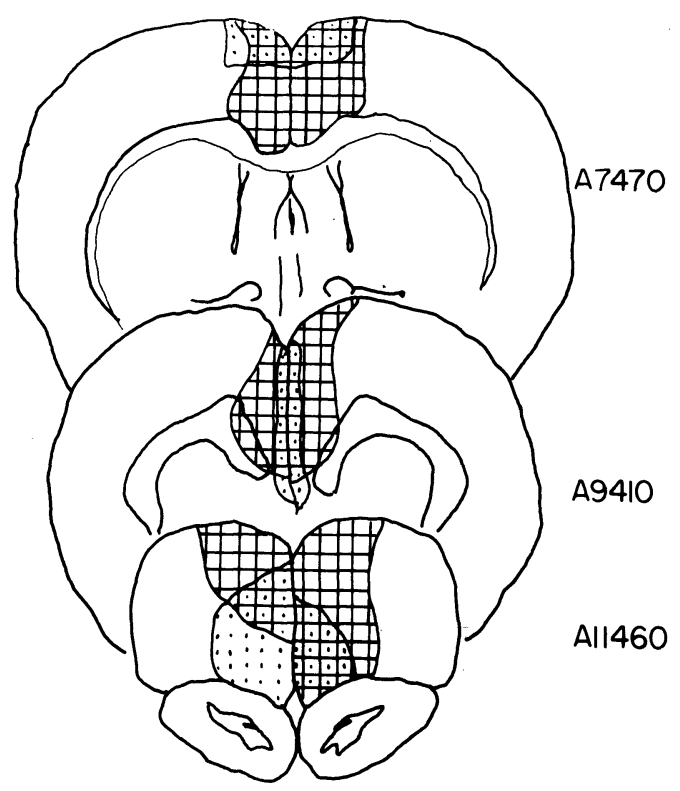

Figure 2. Tracing of the largest and smallest lesions on three coronal sections taken from the labeled drawings in Konig and Klippel (1963). 
behavioral function observed after surgery, it is most evident that the startle reflex in the brain-damaged animals exhibited the usual facilitative effect of moderate increases in background noise level, as well as the probably related effect of this variable on activity measures. Davis and Gendleman (1977) observed that startle reflex facilitation was not obtained after midbrain transection. Their data revealed that a significant portion of the system which produces facilitation is located in the forebrain. Our data rule out any necessary contribution of medial frontal cortex to this system.

\section{REFERENCES}

BARKER, D. J., \& Thomas, G. J. Ablation of cingulate cortex in rats impairs alternation learning and retention. Journal of Comparative and Physiological Psychology, 1965, 60, 353-375.

BRUTKOWSKI, S. Functions of prefrontal cortex in animals. Physiological Reviews, 1965, 45, 721-746.

Clemente, C. D. Forebrain mechanisms related to internal inhibition and sleep. Conditional Reflex, 1968, 3, 145-174.

DAvis, M. Signal-to-noise ratio as a predictor of startle amplitude and habituation in the rat. Journal of Comparative and Physiological Psychology, 1974, 86, 812-825.

Davis, M., \& Gendelman, P. M. Plasticity of the acoustic startle response in the acutely decerebrate rat. Journal of Comparative and Physiological Psychology, 1977, 91, 549-563.

Hammond, G. R. Frontal cortical lesions and prestimulus inhibition of the rat's acoustic startle reaction. Physiological Psychology, 1974, 2, 151-156.

Ison, J. R., \& HAMmond, G. R. Modification of the startle reflex in the rat by changes in the auditory and visual environments. Journal of Comparative and Physiological Psychology, 1971, 75, 435-452.

Ison, J. R., \& LEONARD, D. W. Effects of auditory stimuli on the amplitude of the nictitating membrane of the rabbit (Oryctolagus cuniculus). Journal of Comparative and Physiological Psychology, 1971, 75, 157-164.

König, J. F. R., \& KlipPel, R. A. The rat brain. A stereotaxic atlas of the forebrain and lower parts of the brainstem. Baltimore: Williams and Wilkins, 1963.

LYNCH, G. S. Separable forebrain systems controlling different manifestations of spontaneous activity. Journal of Comparative and Physiological Psychology, 1970, 70, 48-59.

MacMillan, A. St. C., Gray, J. A., \& Ison. J. R. An apparent new instance of stimulus intensity dynamism during discrimination of duration of repeating auditory stimuli. Quarterly Journal of Experimental Psychology, 1973, 25, $62-70$.

Sheard, M. H., Astrachan, D., \& Davis, M. Effect of noise on shock-elicited fighting in rats. Nature, 1975, 257, 43-44.

SKINNER, J. E., \& LiNDSLEY, D. B. Electrophysiological and behavioral effects of blockage of the non-specific thalamocortical system. Brain Research, 1967, 6, 95-118.

Stevens, S. S. Power-group transformations under glare, masking, and recruitment. Journal of the Acoustical Society of America, 1966, 39, 725-735.

VALENSTEIN, E. W. A note on anesthetizing rats and guinea pigs. Journal of the Experimental Analysis of Behavior, 1961, 4, 6.

Velasco, M., \& Lindsley, D. B. Role of orbital cortex in regulation of the thalamo-cortical electrical activity. Science, $1965,149,1375-1377$.

(Received for publication January 5, 1978; accepted January 23, 1978.) 